\title{
Toxoplasmosis aguda diseminada fatal en una paciente adulta inmunocompetente proveniente del Pacífico colombiano
}

\author{
Armando Daniel Cortés ${ }^{1}$, Natalia Aguirre ${ }^{2}$ \\ 1 Facultad de Salud, Departamento de Patología, Universidad del Valle, Cali, Colombia \\ 2 Programa de Anatomía Patológica y Patología Clínica, Universidad del Valle, Cali, Colombia \\ Se presenta el caso de una paciente inmunocompetente de 72 años de edad, proveniente del \\ departamento del Chocó, con un cuadro clínico de 12 días de fiebre, cefalea, deterioro neurológico \\ progresivo y rápida evolución a falla orgánica múltiple y muerte. \\ En el estudio histopatológico de los tejidos obtenidos en la necropsia, se identificaron quistes tisulares \\ morfológicamente sugestivos de ser bradizoítos de Toxoplasma gondii, lo que se confirmó mediante \\ inmunohistoquímica en corazón, cerebro y músculo estriado.
}

Palabras clave: Toxoplasma; toxoplasmosis; miocarditis; Colombia. doi: https://doi.org/10.7705/biomedica.v38i0.4087

\begin{abstract}
Severe disseminated acute toxoplasmosis in an adult immunocompetent patient from the Colombian Pacific

We present the case of a 72-year-old immunocompetent patient from Chocó, Colombia, with a 12-day course of fever, headache, progressive neurological deterioration, and rapid evolution to multiorgan failure and death.

In the histopathological study of tissues obtained at necropsy, tissue cysts morphologically suggestive of being bradyzoites of Toxoplasma gondii were identified and confirmed by immunohistochemistry in heart, brain, and striated muscle.
\end{abstract}

Key words: Toxoplasma; toxoplasmosis; myocarditis; Colombia.

doi: https://doi.org/10.7705/biomedica.v38i0.4087

La toxoplasmosis es una zoonosis parasitaria de distribución mundial. Los seres humanos generalmente se infectan por el consumo de carne o de verduras poco cocidas, o de agua contaminada por heces de gatos. Habitualmente, se presenta de forma asintomática en huéspedes sanos, o produce una enfermedad similar a la mononucleosis infecciosa con adenopatía cervical en el $10 \%$ de los sujetos (1). Los casos graves de toxoplasmosis y las manifestaciones clínicas, como la miocarditis y la encefalitis, son raras y se desarrollan con frecuencia en pacientes inmunocomprometidos $(2,3)$, especialmente en aquellos con sida.

\author{
$\overline{\text { Correspondencia: }}$ \\ Natalia Aguirre, Carrera 60 № 10-31, apartamento 502 D, Unidad \\ Santa Anita, Cali, Colombia \\ Teléfono: (300) 2312381 \\ natav20@hotmail.com
}

Recibido: 26/10/17; aceptado: 30/05/18
Se presenta el caso de una paciente inmunocompetente con un cuadro de deterioro neurológico progresivo y rápida evolución a falla orgánica múltiple y muerte.

\section{Reporte de caso}

Se trata de una paciente de 72 años de edad que fue llevada por sus familiares al servicio de urgencias de un hospital de tercer nivel en Cali (Colombia), por un cuadro clínico de 12 días de evolución consistente en elevación no cuantificada de la temperatura, asociada con cefalea intensa y deterioro neurológico progresivo.

En el momento del ingreso, la paciente no respondía al Ilamado, estaba febril $\left(38,4{ }^{\circ} \mathrm{C}\right)$, con taquicardia (112 latidos por minuto), taquipnea (28 respiraciones por minuto), saturación arterial de oxígeno $\left(\mathrm{SaO}_{2}\right)$ de $86 \%$, movimientos tónicos de los miembros superiores e incontinencia urinaria.

\section{Contribución de los autores:}

Natalia Aguirre: elaboración del informe definitivo de la necropsia clínica de la paciente

Armando Cortés: revisión y dirección de los residentes en el proceso de la realización de las necropsias, revisión de los informes y dictamen del diagnóstico definitivo 
Una hora después del ingreso, presentó falla respiratoria, y requirió intubación orotraqueal y asistencia respiratoria mecánica. Con la impresión diagnóstica de infección del sistema nervioso central, se inició el tratamiento antibiótico con cefepime, vancomicina y ampicilina.

En los exámenes de ingreso se encontró trombocitopenia $\left(29.000 / \mathrm{mm}^{3}\right)$, elevación de la creatinina (3 mg/dl), la deshidrogenasa láctica (985 UI/l) y la creatina-fosfocinasa (CPK) (1.176 UI/L). Los estudios para leptospirosis, malaria, HIV y dengue, fueron negativos, así como la punción lumbar con presión de apertura de $13 \mathrm{~cm} \mathrm{H}_{2} \mathrm{O}$ y pleocitosis a expensas de los neutrófilos $\left(12 / \mathrm{mm}^{3}\right)$; los niveles de proteínas eran de $106 \mathrm{mg} / \mathrm{dl} \mathrm{y}$, los de glucosa, de $68 \mathrm{mg} / \mathrm{dl}$.

En la tomografía computadorizada (TC) de cerebro, se observó hidrocefalia con ventriculomegalia e hipodensidades periventriculares (figura 1) y en la de tórax de alta resolución, un patrón en vidrio esmerilado con atelectasia del lóbulo inferior pulmonar izquierdo (figura 2).

Con base en los hallazgos de las imágenes diagnósticas y el cuadro clínico de la paciente, el grupo de médicos tratantes consideró que se trataba de un posible cuadro de tuberculosis pulmonar y meníngea, por lo que se prescribió, además, el tratamiento tetraconjugado para tuberculosis.

A pesar del tratamiento, la paciente evolucionó rápidamente a una falla orgánica múltiple y falleció a los diez días del ingreso. Se hizo la autopsia y en el estudio histopatológico de tejido miocárdico, cerebral y de músculo esquelético, se encontraron quistes tisulares con formas sugestivas de bradizoítos de Toxoplasma gondii (figuras 3-7). Estos se sometieron al estudio de inmunohistoquímica utilizando el anticuerpo monoclonal específico para la proteína de membrana P30 de $T$. gondii (Novocastra $^{\mathrm{TM}}$, clon TP3), purificado a partir de líquido ascítico de ratón y diluido en una solución tampón de fosfato salino (PBS), pH 7,6, con albúmina de suero bovino (BSA) al $1 \%$ y $0,09 \%$ de azida de sodio, cuyo resultado confirmó la presencia del parásito en los tejidos evaluados (figuras 8-10).

\section{Discusión}

Toxoplasma gondii es un parásito protozoario intracelular obligado, cuyos huéspedes definitivos son los félidos domésticos y salvajes; en ellos se desarrollan las etapas sexuales y asexuales de su ciclo biológico (4). En el humano y en diversos huéspedes intermediarios que ingieren quistes

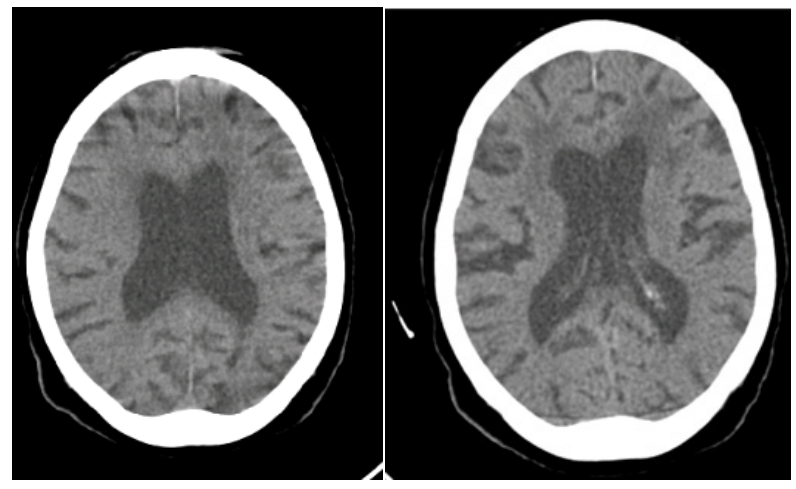

Figura 1. Tomografía computadorizada de cerebro: se observan hidrocefalia e hipodensidades periventriculares fronto-parietales.

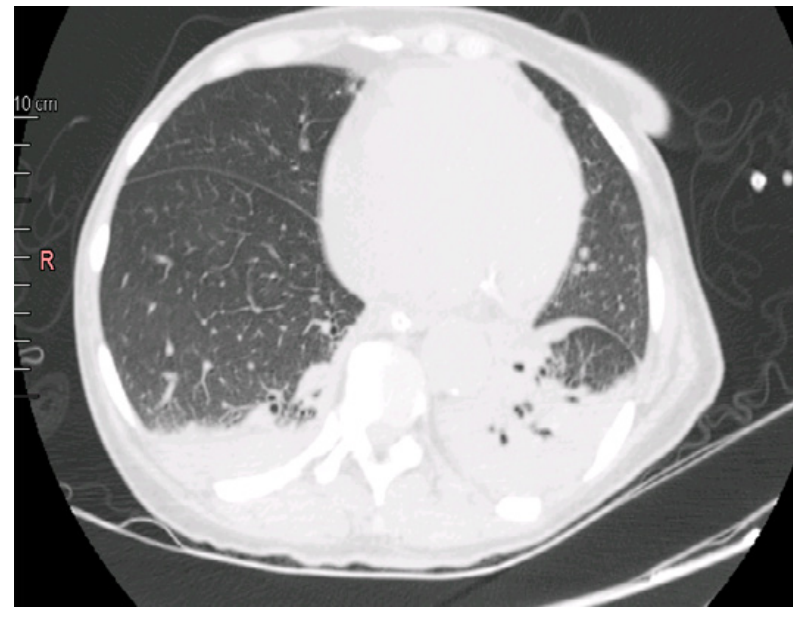

Figura 2. Tomografía computadorizada de tórax: patrón en vidrio esmerilado con atelectasia de lóbulo inferior pulmonar izquierdo

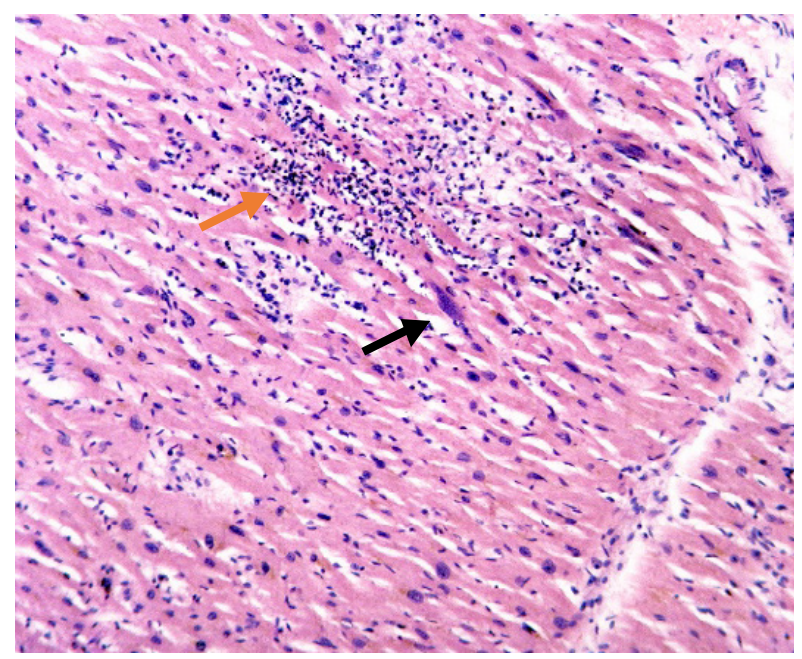

Figura 3. Músculo cardiaco con extenso infiltrado inflamatorio mononuclear (flecha naranja) y un quiste en la fibra muscular con formas sugestivas de bradizoítos (flecha negra). Hematoxilina y eosina, $4 \mathrm{X}$. 
con bradizoítos u ooquistes con esporozoítos, los parásitos invaden las células de la mucosa de las vías digestivas, donde se diferencian en taquizoítos y se multiplican localmente antes de diseminarse por vía sanguínea o linfática. Tienen la capacidad de invadir todas las células nucleadas del cuerpo, aunque los órganos preferidos son los ganglios linfáticos, el cerebro, el corazón y los pulmones (5).

El diagnóstico puede hacerse mediante pruebas serológicas, amplificación de secuencias específicas de ácido nucleico (reacción en cadena de la polimerasa, PCR), demostración histológica del parásito o de sus antígenos (tinción con inmunoperoxidasa) o por aislamiento del organismo. Otros métodos raramente usados incluyen la demostración de antigenemia y del antígeno en suero y fluidos corporales, la prueba cutánea de toxoplasmina y la transformación de linfocitos específicos del antígeno (6).

La prevalencia de la infección por $T$. gondii en Europa ha disminuido en las últimas cuatro décadas. En Francia, por ejemplo, la seroprevalencia general de la infección en mujeres en edad fértil disminuyó de $54 \%$ en 1995 a $37 \%$ en 2010; en los Estados Unidos, es de 15,8 a 19,2 \%; en Suramérica, se presentan tasas mayores que alcanzan el $73 \%$ en Brasil y oscilan entre el 26 y el $77 \%$ en Colombia (7).

En la mayoría de los casos, la infección es asintomática y solo el $10 \%$ de las personas presentan síntomas que, generalmente, corresponden a una afección leve y de resolución espontánea. Los cuadros diseminados y las complicaciones graves de la infección con este parásito, usualmente se describen en personas inmunocomprometidas; sin embargo, desde 1998 se vienen publicando reportes de casos de pacientes inmunocompetentes con cuadros graves.

El estudio de dichos casos ha permitido identificar cepas silvestres de $T$. gondii cuya infección ha originado tasas de mortalidad de hasta $27 \%$ en una de las series (8). Los casos reportados en Guyana Francesa, Brasil y Surinam $(2,8,9)$, evidenciaron que las cepas atípicas podrían presentar una mayor capacidad patógena y, en algunas aisladas en Suramérica, se han identificado genotipos atípicos y una mayor diversidad en comparación con las descritas en Norteamérica y Europa, todas ellas asociadas con cuadros clínicos más graves en ratones y en seres humanos $(10,11)$.

En el 2013, De la Torre A, et al., publicaron un estudio en el que se evaluaron 19 casos de

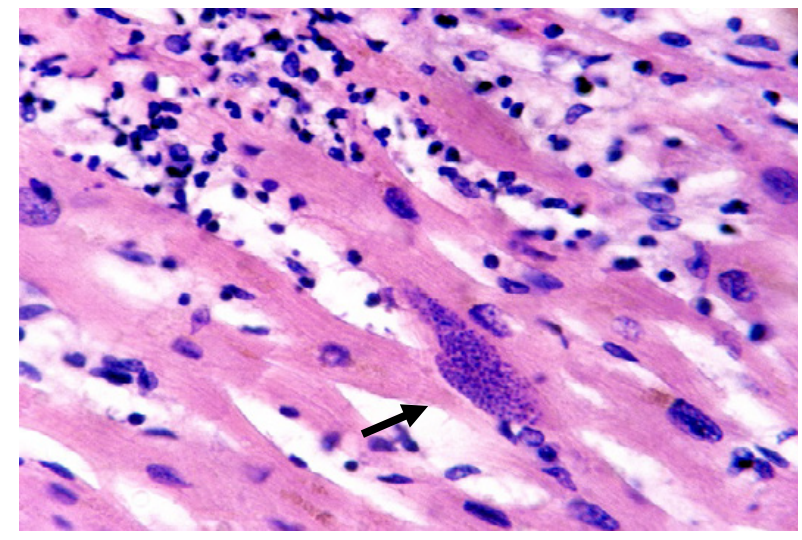

Figura 4. Quiste en músculo cardiaco con formas sugestivas de bradizoítos (flecha). Hematoxilina y eosina, 10X.

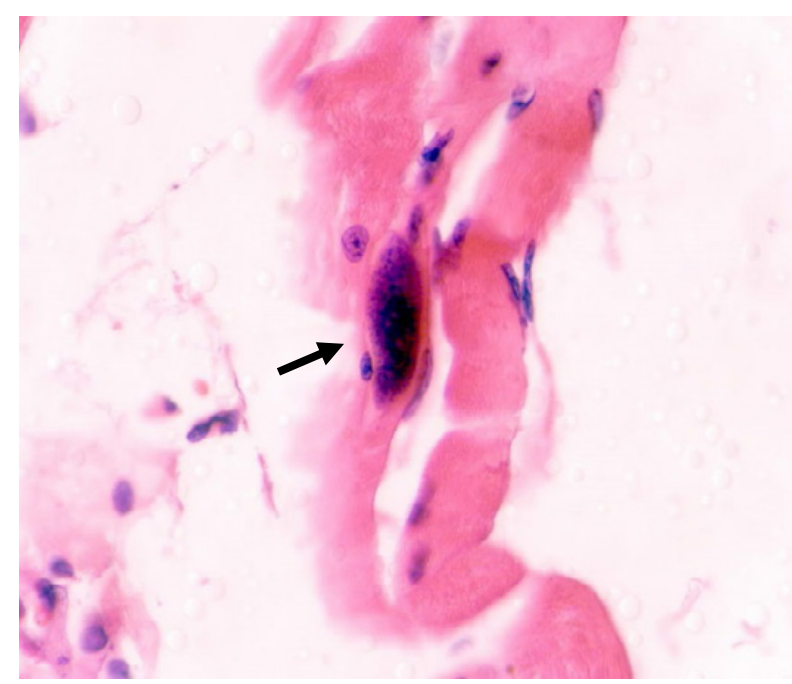

Figura 5. Quiste en músculo estriado con formas sugestivas de bradizoítos (flecha). Hematoxilina y eosina, 10X.

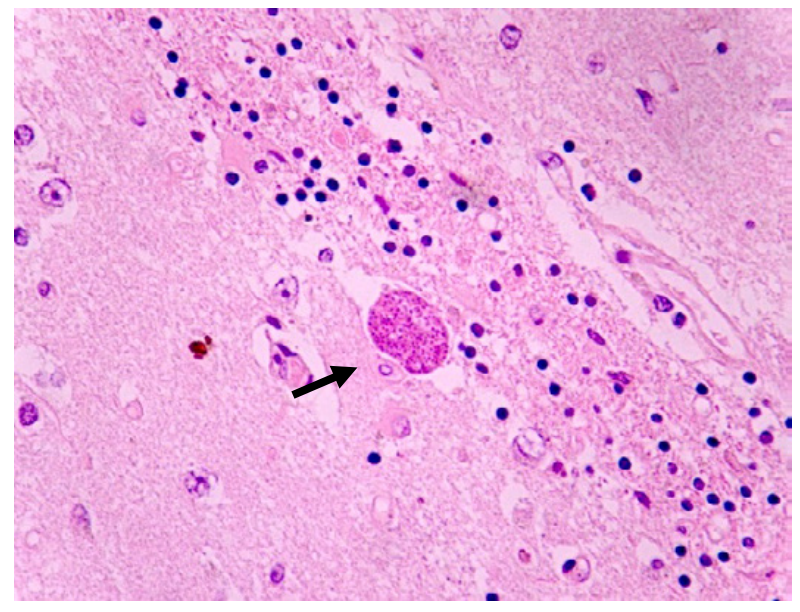

Figura 6. Tejido cerebral con necrosis focal y quiste con formas sugestivas de bradizoítos (flecha). Hematoxilina y eosina, 40X. 


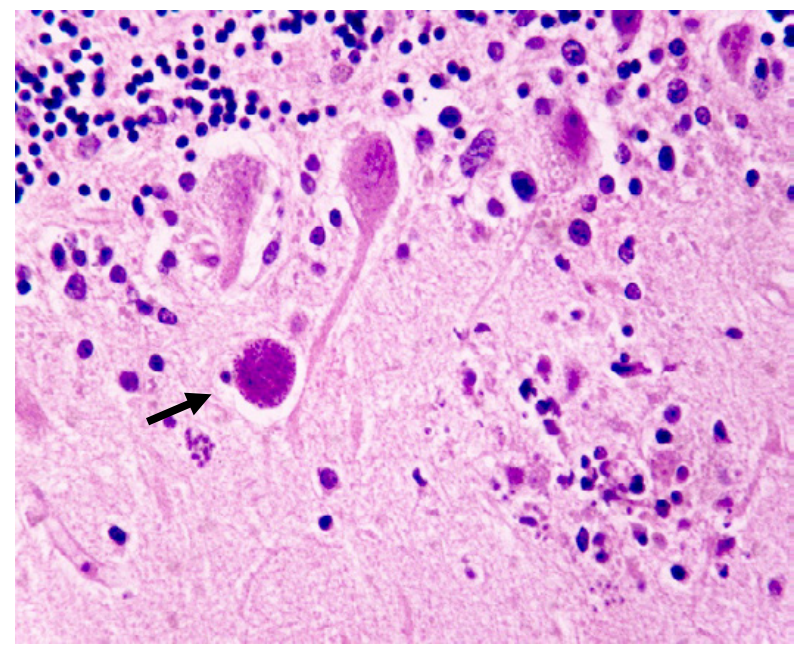

Figura 7. Corte histopatológico de cerebelo con quiste que contiene formas sugestivas de bradizoítos (flecha). Hematoxilina y eosina, 40X.

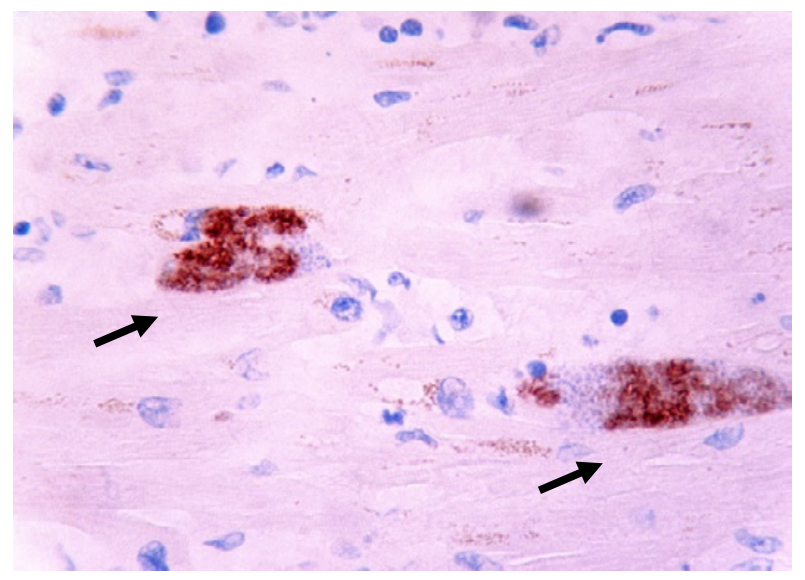

Figura 8. Estudio de inmunohistoquímica positivo para Toxoplasma gondii en músculo cardiaco (flecha), 40X

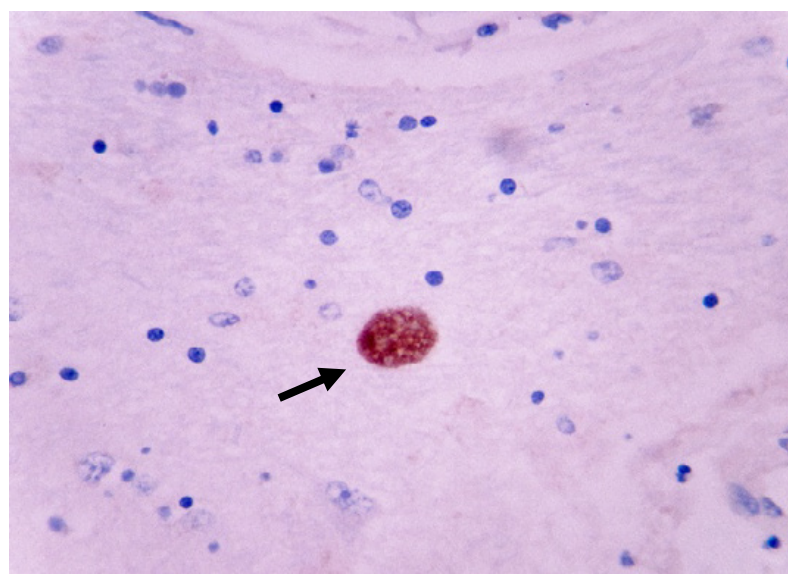

Figura 9. Estudio de inmunohistoquímica positivo para Toxoplasma gondii en cerebro (flecha). 40X pacientes franceses y 23 casos de colombianos con toxoplasmosis ocular activa confirmada, cuyas reacciones clínicas, parasitológicas e inmunológicas se compararon y se relacionaron con las cepas. Los autores encontraron que los pacientes colombianos poseían serotipos atípicos heterogéneos, en tanto que en los franceses la reacción a los péptidos de la cepa de tipo II era uniforme y que, en cambio, los patrones de proteína reconocidos por los anticuerpos oculares y los de las citocinas eran diferentes entre las dos poblaciones. En los pacientes franceses, se observó una gran respuesta inflamatoria con incremento de los niveles de IL-6, IL-1 $\beta$, IL-8, MIP-1 $\beta$, MCP-1 y G-CSF, en tanto que en los pacientes colombianos se registró una baja expresión de citocinas proinflamatorias incluidas las IFN- $\gamma$, IL-15, IL-17, IL-2, IL-10, MIP$1 \beta$, GM-CSF y G-CSF, con la excepción de los niveles elevados de TNF- $\alpha$ e IL-6 (12).

En junio del 2009, Pino, et al., reportaron un brote epidémico causado por $T$. gondii en personal militar que desarrollaba operaciones en el área de la serranía de La Macarena (Meta). De los 19 casos sospechosos, solo uno fue positivo para el HIV y el resto no presentaba inmunodeficiencia. En estos pacientes, predominó el compromiso del sistema reticuloendotelial evidenciado por la presencia de adenomegalias superficiales ( $100 \%$ de los casos). Debe anotarse que en dicho brote de toxoplasmosis aguda hubo un alto porcentaje de compromiso pulmonar, gastrointestinal e, incluso, un caso grave de compromiso del miopericardio (13).

La paciente cuyo caso se presenta era una ama de casa previamente sana de 72 años de edad, cuya historia clínica, confirmada posteriormente por la familia, no registraba antecedentes ni evidencia

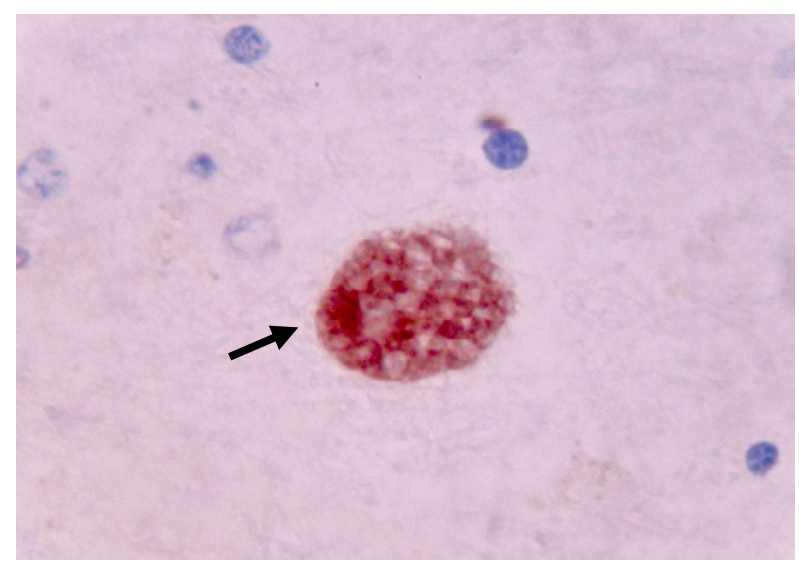

Figura 10. Estudio de inmunohistoquímica positivo para Toxoplasma gondii en cerebro. 100X 
de infecciones recurrentes, como tampoco enfermedades metabólicas ni exposición a tratamientos inmunosupresores $u$ otros factores de riesgo para el desarrollo de una inmunodeficiencia secundaria que facilitara la infección y la progresión de la enfermedad tal como sucedió (14).

Provenía de la vereda de Fugiado, municipio de Istmina, en el departamento del Chocó, región selvática del Pacífico colombiano localizada sobre la cuenca de los ríos Atrato y San Juan, de difícil acceso, donde no se cuenta con agua potable y no hay una adecuada manipulación y cocción de los alimentos, aspectos que, sumados al consumo de carne de animales selváticos, podrían ser factores de riesgo para la transmisión de este parásito y el desarrollo de una infección que terminó en la muerte de la paciente.

No se detectaron cuadros clínicos similares en los familiares ni en otros miembros de la comunidad. No obstante, sería importante estudiar el lugar para la eventual detección de otros casos, pues este es el primero de toxoplasmosis aguda diseminada reportado en una paciente inmunocompetente en la región del Pacífico colombiano, el cual se explicaría por el desconocimiento de las nuevas cepas atípicas y su efecto en pacientes inmunocompetentes, así como por su rápida evolución y por el hecho de que no se indagó suficientemente sobre los factores de riesgo y ello impidió que los médicos tratantes estuvieran alertas frente a la enfermedad.

Por ello, es importante conocer y estudiar este tipo de casos, pues el parásito puede contarse entre los nuevos genotipos que se investigan actualmente, y cuya diversidad y recombinación genética están generando nuevos mecanismos patogénicos (15). Esto permitiría establecer medidas de detección y tratamiento oportunas, así como estrategias de prevención en estas zonas de alto riesgo.

\section{Consideraciones éticas}

Se contó con la autorización de la familia de la paciente para publicar el caso.

\section{Conflicto de intereses}

Los autores declaran no tener conflicto de intereses.

\section{Financiación}

No se recibió financiación alguna.

\section{Referencias}

1. Sobanski V, Ajzenberg D, Delhaes L, Bautin N, Just $\mathrm{N}$. Severe toxoplasmosis in immunocompetent hosts: $\mathrm{Be}$ aware of atypical strains. Am J Respir Crit Care Med. 2013; 187:1143-5. https://doi.org/10.1164/rccm.201209-1635LE
2. Carme B, Bissuel F, Ajzenberg D, Bouyne R, Aznar C, Demar $\mathbf{M}$, et al. Severe acquired toxoplasmosis in immunocompetent adult patients in French Guiana. J Clin Microbiol. 2002;40:4037-44. https://doi.org/10.1128/JCM.40. 11.4037-4044.2002

3. Montoya J, Liesenfeld O. Toxoplasmosis. Lancet. 2004;363: 1965-76. https://doi.org/10.1016/S0140-6736(04)16412-X

4. Saadatnia G, Golkar M. A review on human toxoplasmosis. Scand J Infect Dis. 2012;44:805-14. https://doi.org/10.3109 /00365548.2012.693197

5. Nunura J, Endo S, Salazar D, Rodríguez A, Pereyra $\mathbf{S}$, Solís $\mathbf{H}$, et al. Disseminated toxoplasmosis in an immunocompetent patient from Peruvian Amazon. Rev Inst Med Trop Sao Paulo. 2010;52:107-10. https://doi. org/10.1590/S0036-46652010000200008

6. Montoya J. Laboratory diagnosis of Toxoplasma gondii infection and toxoplasmosis. J Infect Dis. 2002;185(Suppl.1): S73-82. https://doi.org/10.1086/338827

7. Petersen E, Ajzenberg D, Mandelbrot L, Gómez-Marín JE. Protozoan diseases: Toxoplasmosis. International Encyclopedia of Public Health. Second edition. Elsevier; 2017. p. 114-32. https://doi.org/10.1016/B978-0-12-803678-5.00361-1

8. Demar M, Ajzenberg D, Maubon D, Djossou F, Panchoe D, Punwasi W, et al. Fatal outbreak of human toxoplasmosis along the Maroni River: Epidemiological, clinical, and parasitological aspects. Clin Infect Dis. 2007;45:88-95. https://doi.org/10.1086/521246

9. Leal F, Cavazzana C, Franco De Andrade H, Galisteo AJ Jr, de Mendonça JS, Kallas EG. Toxoplasma gondii pneumonia in immunocompetent subjects: Case report and review. Clin Infect Dis. 2007;44:e62-6. https://doi.org/10. 1086/511871

10. Demar M, Hommel D, Djossou F, Peneau C, Boukhari R, Louvel D, et al. Acute toxoplasmoses in immunocompetent patients hospitalized in an intensive care unit in French Guiana. Clin Microbiol Infect. 2012;18:E221-31. https://doi. org/10.1111/j.1469-0691.2011.03648.x

11. Pena HF, Gennari SM, Dubey JP, Su C. Population structure and mouse-virulence of Toxoplasma gondii in Brazil. Int J Parasitol. 2008;38:561-9. https://doi.org/10. 1016/j.ijpara.2007.09.004

12. De la Torre A, Sauer A, Bourcier T, Speeg-Schatz C, Ballonzoli L, Ajzenberg D, et al. Severe South American ocular toxoplasmosis is associated with decreased IFNgamma/IL-17A and increased IL-6/IL-13 intraocular levels. PLoS Neglected Trop Dis. 2013;7:e2541. https://doi.org/10. 1371/journal.pntd.0002541

13. Pino L, Salinas J, López M. Description of an epidemic outbreak of acute toxoplasmosis in immunocompetent patients from Colombian Armed Forces during jungle operations. Infectio. 2009;13:83-91. https://doi.org/10.1016/ S0123-9392(09)70729-5

14. Zea F. Inmunocompentencia en adultos: más que VIH negativo. Colombia Médica. 2016;47:176. https://www.redalyc. org/pdf/283/28348402007.pdf

15. Darde ML. Toxoplasma gondii, "new" genotypes and virulence. Parasite. 2008;15:366-71. https://doi.org/10.1051/ parasite/2008153366 